\title{
Outpatient management without initial assessment for febrile patients undergoing adjuvant chemotherapy for breast cancer
}

\author{
KOSEI KIMURA ${ }^{1}$, SATORU TANAKA ${ }^{2}$, MITSUHIKO IWAMOTO ${ }^{1}$, HIROYA FUJIOKA ${ }^{1}$, \\ NAYUKO SATO $^{1}$, RISA TERASAWA ${ }^{1}$, KANAKO KAWAGUCHI ${ }^{1}$, JUNNA MATSUDA ${ }^{1}$, \\ NODOKA UMEZAKI $^{1}$ and KAZUHISA UCHIYAMA ${ }^{1}$ \\ ${ }^{1}$ Department of Breast and Endocrine Surgery, Osaka Medical College Hospital, Takatsuki, Osaka 569-8686; \\ ${ }^{2}$ Department of Breast Surgery, National Hospital Organization Osaka-Minami Medical Center, \\ Kawachinagano, Osaka 586-8521, Japan
}

Received March 1, 2016; Accepted July 25, 2016

DOI: $10.3892 / \mathrm{mco} .2016 .992$

\begin{abstract}
The purpose of this study was to retrospectively analyze the feasibility of outpatient management without initial assessment for febrile patients undergoing adjuvant chemotherapy for breast cancer. A total of 131 consecutive patients with breast cancer treated with adjuvant or neoadjuvant chemotherapy from 2011 to 2013 at Osaka Medical College Hospital (Osaka, Japan) were retrospectively reviewed. In the case of developing a fever (body temperature, $\geq 38^{\circ} \mathrm{C}$ ), the outpatients had been instructed to take previously prescribed oral antibiotics for 3 days without any initial assessment, and if no improvement had occurred by then, they were required to visit the hospital for examination and to undergo treatment based on the results of a risk assessment for complications. The primary aim of the present study was to assess the outcome of febrile episodes, while the secondary aim was to assess the incidence of febrile episodes, hospitalizations, and the type of chemotherapy. The 131 patients received 840 chemotherapy administrations. Fifty-five patients $(42.0 \%)$ had a total of 75 febrile episodes after 840 chemotherapy administrations (8.9\%). Treatment failure occurred in 12 of the 75 episodes (16.0\%) in 11 of the 55 patients (20.0\%). Only four episodes required hospitalization. Treatment success was achieved in 63 episodes (84.0\%). In conclusion, the feasibility of outpatient management without initial assessment was evaluated in the present study for febrile patients undergoing adjuvant chemotherapy for breast cancer, and the outpatient strategy regimen may be safe and convenient for these patients.
\end{abstract}

Correspondence to: Dr Kosei Kimura, Department of Breast and Endocrine Surgery, Osaka Medical College Hospital, 2-7 Daigaku-Machi, Takatsuki, Osaka 569-8686, Japan

E-mail: sur121@osaka-med.ac.jp

Key words: febrile neutropenia, breast cancer, adjuvant chemotherapy, oral antibiotics, outpatient management

\section{Introduction}

Chemotherapy-induced neutropenia is the most common type of toxicity associated with the administration of anti-cancer drugs. In a large prospective registry, $37 \%$ of patients with breast cancer developed an absolute neutrophil count (ANC) of $<500$ cells/ $\mu l$ during the first four cycles of treatment (1).

All patients with chemotherapy-induced neutropenia are at risk of developing febrile neutropenia (FN), which is the first manifestation of life-threatening bacterial infection (2). According to the Infectious Diseases Society of America guidelines, $\mathrm{FN}$ is defined as an oral temperature of $\geq 38^{\circ} \mathrm{C}$ for $1 \mathrm{~h}$ with either an ANC of $<500$ cells $/ \mu \mathrm{l}$ or an ANC that is expected to decrease to $<500$ cells $/ \mu$ l within $48 \mathrm{~h}$ (3). However, the Japanese Society of Medical Oncology (JSMO) guidelines define $\mathrm{FN}$ as a single axillary temperature of $\geq 37.5^{\circ} \mathrm{C}$ and an ANC of $<1,000$ cells $/ \mu 1$ with a predicted decline to $<500$ cells $/ \mu$ l within $48 \mathrm{~h}(4,5)$.

Several guidelines recommend that febrile patients should go to the hospital immediately for initial assessment including an interview, clinical examination and laboratory evaluation $(6,7)$. Once $\mathrm{FN}$ is diagnosed, the affected patient should receive anti-bacterial treatment according to the results of a risk assessment such as that performed using the Multinational Association for Supportive Care in Cancer (MASCC) score (8).

Although standard treatment of FN involves the intravenous administration of anti-bacterial agents in the hospital setting, low-risk patients (i.e. those in an overall good condition) can be safely managed at home with oral antibiotics $(6,9)$. Mizuno et al (10) recently evaluated the feasibility of risk assessment over the telephone in the outpatient management of patients with low-risk FN who do not visit the hospital. They concluded that telephone-based risk assessment of patients with FN may be convenient.

Most patients with breast cancer undergoing adjuvant or neoadjuvant chemotherapy are thought to have controlled cancer and a good performance status, and if they have been diagnosed with FN, they are likely to be stratified as low-risk patients. For this reason, initial assessment at the hospital or over the telephone may not be required. Although most 
patients with a single axillary body temperature of $\geq 38^{\circ} \mathrm{C}$ occurring between days 8 and 14 after the start of chemotherapy may have neutropenia and may be diagnosed with FN (10), outpatient management without initial assessment for these patients is routinely performed at our hospital. Implementation of this type of outpatient management can avoid unnecessary consultations, decrease costs, limit the risk of hospital-acquired infections and improve patients' quality of life (11-13), although it can also lead to insufficient initial assessment and inappropriate initial therapy. The JSMO guidelines do not recommend this type of outpatient management without initial assessment for febrile patients, although they also state that these recommendations may be modified, depending on the characteristics of individual malignancies and organs (5).

The present study has retrospectively analyzed the feasibility of outpatient management without initial assessment for febrile patients undergoing adjuvant or neoadjuvant chemotherapy for breast cancer.

\section{Materials and methods}

Study design. A total of 131 consecutive patients with breast cancer undergoing adjuvant or neoadjuvant chemotherapy between January 2011 and December 2013 at Osaka Medical College Hospital (Osaka, Japan) were retrospectively reviewed. The following data were collected from the patients' medical records: Age, cancer stage, estrogen receptor status, human epidermal growth factor receptor 2 status, comorbidities, chemotherapy regimen, dose intensity, febrile episodes, use of granulocyte colony-stimulating factor (G-CSF), use of antibiotics, hospital attendance, hospital admission and outcome. Patients with metastatic breast cancer were excluded. A febrile episode was defined as a single axillary temperature of $\geq 38^{\circ} \mathrm{C}$ occurring between days 8 and 14 after the start of chemotherapy.

All patients had been instructed, orally and in writing, to take previously prescribed antibiotics (ciprofloxacin $200 \mathrm{mg}$ three times daily in 2011 or levofloxacin $500 \mathrm{mg}$ once daily from 2012) for 3 days if their temperature increased to $>38^{\circ} \mathrm{C}$, and to visit the hospital if no improvement was apparent after 3 days of antibiotic therapy. Following an examination at the hospital, the patients received the same or a different anti-bacterial treatment (cefepime or meropenem) on either an outpatient or an inpatient basis, according to the results of a risk assessment for complications based on the MASCC score (8).

Adjuvant and neoadjuvant chemotherapy regimens for the patients enrolled in the present study included the following: 5-Fluorouracil $500 \mathrm{mg} / \mathrm{m}^{2}$, epirubicin $100 \mathrm{mg} / \mathrm{m}^{2}$ and cyclophosphamide $500 \mathrm{mg} / \mathrm{m}^{2}$ every 3 weeks for four or six cycles (FEC); FEC for four cycles followed by docetaxel (DOC) 75 or $100 \mathrm{mg} / \mathrm{m}^{2}$ with optional trastuzumab (Tr) $6 \mathrm{mg} / \mathrm{kg}(8 \mathrm{mg} / \mathrm{kg}$ as loading dose) every 3 weeks for four cycles (FEC-DOC \pm Tr); FEC for four cycles followed by nanoparticle albumin-bound (nab)-paclitaxel $260 \mathrm{mg} / \mathrm{m}^{2}$ with optional Tr every 3 weeks for four cycles (FEC-nab-PTX \pm Tr); DOC $75 \mathrm{mg} / \mathrm{m}^{2}$ and cyclophosphamide $600 \mathrm{mg} / \mathrm{m}^{2}$ every 3 weeks for four cycles (TC); and DOC $75 \mathrm{mg} / \mathrm{m}^{2}$, carboplatin AUC6 and Tr every 3 weeks for six cycles $(\mathrm{TCH})$.
Table I. Characteristics of the study population $(n=131)$.

\begin{tabular}{lc}
\hline Characteristic & Patients, $\mathrm{n}(\%)$ \\
\hline Age (years) & 54 \\
Median & $25-77$ \\
Range & \\
Stage & $25(19.1)$ \\
I & $84(64.1)$ \\
II & $22(16.8)$ \\
III & \\
ER/HER2 status & $60(45.8)$ \\
ER(+)/HER2(-) & $26(19.8)$ \\
ER(+)/HER2(+) & $18(13.7)$ \\
ER(-)/HER2(+) & $27(20.6)$ \\
ER(-)/HER2(-) & \\
Charlson comorbidity index & $120(91.6)$ \\
0 & $10(7.6)$ \\
$1 / 2$ & $1(0.8)$ \\
$3 / 4$ & $0(0.0)$ \\
$\geq 5$ &
\end{tabular}

ER, estrogen receptor; HER2, human epidermal growth factor 2; G-CSF, granulocyte colony-stimulating factor.

The use of G-CSF was avoided as much as possible while patients were at low risk, but it was immediately administered if a patient was considered to have become high risk, e.g. due to low performance status or dehydration. The present study was approved by the Medical Ethics Committee of Osaka Medical College Hospital (Osaka, Japan) and all patients provided written informed consent to participate.

Study outcomes. The primary aim of the present study was to assess the outcome of febrile episodes (treatment success and failure rates). The secondary aim was to assess parameters including hospitalizations and the type of chemotherapy. Treatment success was defined as the fever having recovered within 3 days with oral antibiotic therapy. If patients did not receive the oral antibiotic therapy, the patients whose fever did recover without antibiotic therapy within 3 days were also considered to have been successfully treated.

Adverse events were graded according to the National Cancer Institute Common Terminology Criteria for Adverse Events (version 4.0; http://ctep.cancer.gov/ protocolDevelopment/electronic_applications/ctc.htm).

\section{Results}

Patient characteristics. In the present study, a retrospective review of 131 consecutive patients with breast cancer undergoing adjuvant or neoadjuvant chemotherapy between January 2011 and December 2013 at Osaka Medical College Hospital (Osaka, Japan) was performed. The clinical characteristics of these patients are shown in Table I. All patients were female, with a median age of 54 years (range, 25-77 years). Of these patients, only one $(0.8 \%)$ had a Charlson Comorbidity 
Table II. Type of adjuvant or neoadjuvant chemotherapy.

\begin{tabular}{|c|c|c|c|}
\hline Chemotherapy & Patients, n (\%) & $\begin{array}{l}\text { Administrations } \\
\text { planned }(\mathrm{n})\end{array}$ & $\begin{array}{c}\text { Administrations } \\
\text { delivered, } \mathrm{n} \text { (\% of planned) }\end{array}$ \\
\hline Adjuvant & $71(54.2)$ & & \\
\hline Neoadjuvant & $60(45.8)$ & & \\
\hline \multicolumn{4}{|c|}{ Chemotherapy regimen } \\
\hline FEC & $17(13.0)$ & 78 & $63(80.8)$ \\
\hline FEC-DOC & $60(45.8)$ & 480 & $474(98.8)$ \\
\hline FEC-DOC+Tr & $10(7.6)$ & 80 & $77(96.3)$ \\
\hline FEC-nab-PTX & $2(1.5)$ & 16 & $15(93.8)$ \\
\hline FEC-nab-PTX+Tr & $10(7.6)$ & 80 & $80(100.0)$ \\
\hline $\mathrm{TC}$ & $21(16.0)$ & 84 & 77 (91.7) \\
\hline $\mathrm{TC}+\mathrm{Tr}$ & $4(3.1)$ & 16 & $16(100.0)$ \\
\hline $\mathrm{TCH}$ & $5(3.8)$ & 30 & $30(100.0)$ \\
\hline $\mathrm{DOC}+\mathrm{Tr}$ & $2(1.5)$ & 8 & $8(100.0)$ \\
\hline Total & $131(100.0)$ & 872 & $840(96.3)$ \\
\hline
\end{tabular}

FEC, epirubicin + 5-fluorouracil + cyclophosphamide; DOC, docetaxel; Tr, trastuzumab; nab-PTX, nanoparticle albumin-bound paclitaxel; $\mathrm{TC}, \mathrm{DOC}+$ cyclophosphamide; TCH, DOC + carboplatin $+\mathrm{Tr}$.

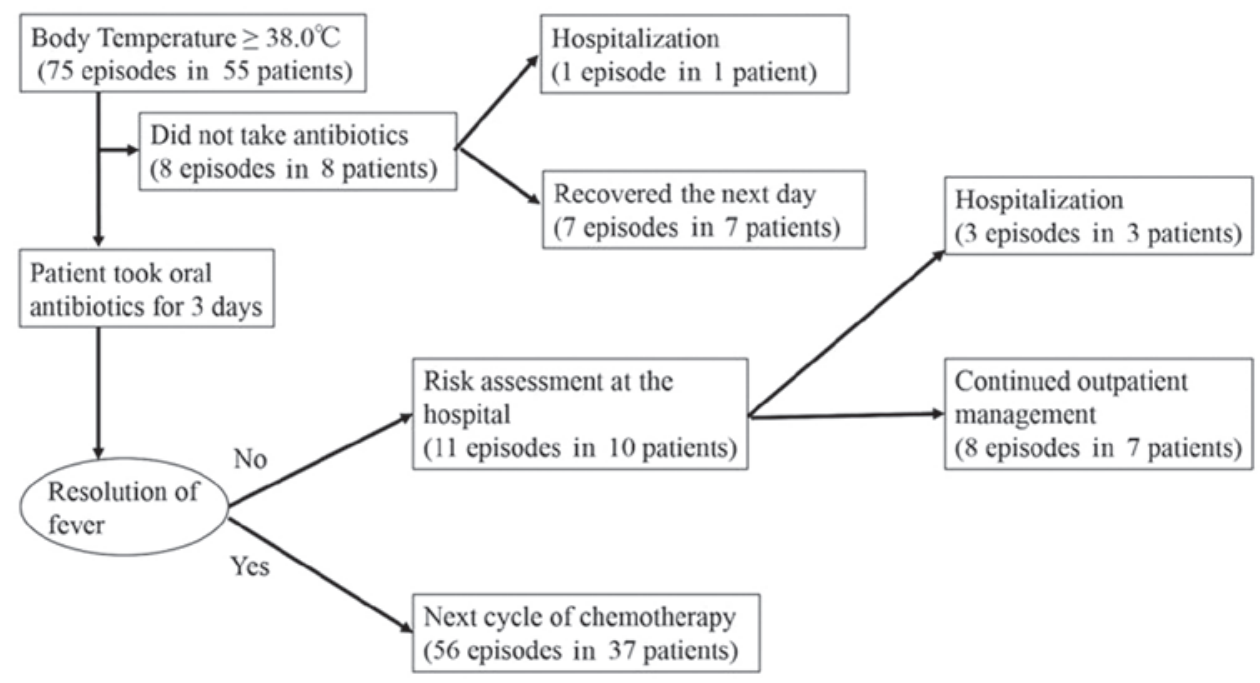

Figure 1. Incidence and outcome of febrile episodes.

Index of 3 (chronic renal failure and chronic hepatitis) (14). None of the patients received primary G-CSF or antibiotic prophylaxis.

Type of chemotherapy. Chemotherapy was administered 840 times among the 131 patients of the present study; 71 patients $(54.2 \%)$ received adjuvant chemotherapy and $60(45.8 \%)$ received neoadjuvant chemotherapy. The chemotherapy regimens applied are listed in Table II. Although 872 administrations were initially planned, this number decreased to 840 (96.3\%) for various reasons.

Incidence of febrile episodes and chemotherapy regimens. Among the 131 patients who received 840 chemotherapy administrations, 55 patients $(42.0 \%)$ had a total of 75 febrile episodes
(8.9\%) (Fig. 1). Thirty-five episodes (46.7\%) occurred in the first chemotherapy cycle. Although all patients were instructed orally and in writing how to take oral antibiotics in this study, 8 (14.5\%) patients did not take these antibiotics despite their febrile episodes. Seven of these eight patients became afebrile the following day without antibiotic therapy. The remaining patient visited the hospital due to general fatigue and required inpatient management. The incidence of febrile episodes for each chemotherapy regimen is summarized in Table III.

Outcome of febrile episodes. Among the 75 febrile episodes in 55 patients, treatment failure occurred in 12 of the episodes $(16.0 \%)$ in 11 patients $(20.0 \%)$ (Table IV). Among eleven of the twelve episodes with treatment failure, grade- 3 or -4 neutropenia was observed (one grade-3 and ten grade-4). 
Table III. Incidence of febrile episodes and chemotherapy regimens (840 administrations among 131 patients).

\begin{tabular}{|c|c|c|}
\hline Fever/chemotherapy & Episodes, n (\%) & Patients, n (\% \\
\hline \multicolumn{3}{|l|}{ Incidence of febrile episodes } \\
\hline Total & $75(8.9)$ & $55(42.0)$ \\
\hline In cycle 1 & $35(46.7)$ & \\
\hline After cycle 1 & $40(53.3)$ & \\
\hline \multicolumn{3}{|l|}{ Chemotherapy regimens } \\
\hline \multicolumn{3}{|l|}{ ( $\mathrm{n}$ administrations for $\mathrm{n}$ patients) } \\
\hline FEC (63 for 17$)$ & $8(12.7)$ & $5(29.4)$ \\
\hline FEC-DOC (474 for 60) & $42(8.9)$ & $30(50.0)$ \\
\hline FEC-DOC + Tr $(77$ for 10$)$ & $4(5.2)$ & $3(30.0)$ \\
\hline FEC-nab-PTX (15 for 2) & $0(0.0)$ & $0(0.0)$ \\
\hline FEC-nab-PTX + Tr (80 for 10) & $4(5.0)$ & $4(40.0)$ \\
\hline $\mathrm{TC}(77$ for 21$)$ & $10(13.0)$ & $8(38.1)$ \\
\hline $\mathrm{TC}+\operatorname{Tr}(16$ for 4$)$ & $4(25.0)$ & $2(50.0)$ \\
\hline $\mathrm{TCH}(30$ for 5$)$ & $2(6.7)$ & $2(40.0)$ \\
\hline $\mathrm{DOC}+\operatorname{Tr}(8$ for 2$)$ & $1(12.5)$ & $1(50.0)$ \\
\hline \multicolumn{3}{|l|}{ Received antibiotics } \\
\hline Yes & & $47(85.5)$ \\
\hline No & & $8(14.5)$ \\
\hline
\end{tabular}

FEC, epirubicin + 5-fluorouracil + cyclophosphamide; DOC, docetaxel; Tr, trastuzumab; nab-PTX, nanoparticle albumin-bound paclitaxel; $\mathrm{TC}, \mathrm{DOC}+$ cyclophosphamide; TCH, DOC + carboplatin $+\mathrm{Tr}$.

Only one of the 11 patients who experienced treatment failure had two episodes, and the two episodes that occurred in this patient were grade-4 neutropenia. Four episodes (including one in a patient with a Charlson Comorbidity Index of 3) required hospitalization due to a high risk of complications based on the MASCC score, and the respective patients were administered G-CSF and intravenous antibiotics. Of these four episodes, one required treatment with intravenously administered antibiotics, while the patient was hospitalized for $\sim 2$ weeks due to phlegmon at the surgical site caused by quinolone-resistant Escherichia (E.) coli. The remaining eight episodes were managed with oral antibiotics on an outpatient basis. Six of these eight episodes were resolved on the following day (total of 4 days of oral antibiotic therapy). Finally, treatment success was achieved in 63 episodes $(84.0 \%)$ among patients who took oral antibiotics and 71 episodes $(94.7 \%)$ among patients who recovered without hospitalization (Fig. 1). No serious adverse events, including mortality, were observed.

\section{Discussion}

In the present study, the feasibility of outpatient management without initial assessment for febrile patients undergoing adjuvant chemotherapy for breast cancer was evaluated. The success rate of the treatment was $84 \%$ (63 of 75 episodes), and 71 of 75 episodes (95\%) were resolved without hospitalization.

Several studies evaluating the use of oral antibiotics in the outpatient management of low-risk FN have reported treat- ment success rates of $77-95 \%(10,14-17)$, which is consistent with the results of the present study.

In the present study, $84 \%$ of the febrile episodes were resolved within 3 days and $92 \%$ were resolved by day 4 . A previous study reported that the median time to defervescence was 2 days (range, 2-4 days) with levofloxacin treatment (14), which is consistent with the results of the present study.

FN is associated with chemotherapy dose reductions or delays in the administration of the chemotherapy that may lead to poor survival of patients with breast cancer $(18,19)$, and the outpatient management performed in the present study was able to maintain the optimal dose intensity (96.3\%).

However, the outpatient management for febrile patients described in the present study was associated with two major problems. First, despite their febrile status, certain patients did not follow the instruction to take their previously prescribed oral antibiotics. In the present study, $14.5 \%$ of patients did not take their antibiotics when febrile episodes occurred. However, most of these patients were likely to have been low-risk patients who recovered without antibiotic therapy. The American Society of Clinical Oncology guidelines recommend that a caregiver is involved in the outpatient management of FN, which may be essential for maintaining optimal adherence to medication (6). The second problem is the development of quinolone-resistant infections. One patient in the present study developed a quinolone-resistant $E$. coli infection and required intravenous antibiotics while hospitalized for $\sim 2$ weeks. Quinolone-resistant E. coli infection is rapidly spreading worldwide, with a prevalence in Japan of almost $20 \%$ (20). As the outpatient management performed in the present study did 
Table IV. Outcome of febrile episodes $(n=75)$.

\begin{tabular}{lc}
\hline Parameter & Episodes, $\mathrm{n}(\%)$ \\
\hline Age (years) & \\
Median & 47 \\
Range & $29-70$ \\
Treatment success & $63(84.0)$ \\
Treatment failure & $12(16.0)$ \\
ANC & \\
G1 & $1(1.3)$ \\
G2 & $0(0.0)$ \\
G3 & $1(1.3)$ \\
G4 & $10(13.3)$ \\
Hospitalization & \\
Yes & $4(5.3)$ \\
No & $8(10.7)$ \\
MASCC score & \\
$>20$ & $8(10.7)$ \\
$\leq 20$ & $4(5.3)$ \\
Charlson comorbidity index & \\
0 & $10(13.3)$ \\
$1 / 2$ & $1(1.3)$ \\
$3 / 4$ & $1(1.3)$ \\
\hline
\end{tabular}

ANC, absolute neutrophil count; MASCC, Multinational Association for Supportive Care in Cancer.

not include any initial assessment, accurate assessment should be performed immediately if a febrile patient does not recover after 3 days of oral antibiotic therapy.

The present study had certain limitations. First, retrospective data from only one institute were used, and the relatively small number of patients may have reduced the statistical power of the conclusions. Furthermore, it has been reported that patients with FN eligible for outpatient management should ideally live $<1 \mathrm{~h}$ from the hospital (6). While most of the patients enrolled in the present study were able to get to our hospital within $1 \mathrm{~h}$, application of this management strategy to institutions for which this is not a given must be done with caution. Furthermore, the incidence of FN among febrile patients in the present study was not clear, as no initial assessment, including laboratory testing for parameters such as the ANC, was performed. Although 55 of the 133 patients in the present study (42.0\%) had a total of 75 febrile episodes after 840 chemotherapy administrations (8.9\%), certain patients may have had a fever without neutropenia. However, the majority of the febrile patients were likely to have had neutropenia, as a febrile episode was defined in the present study as a single axillary temperature of $\geq 38^{\circ} \mathrm{C}$ occurring between days 8 and 14 after the start of chemotherapy. Mizuno et al (10), who evaluated the feasibility of risk assessment over the telephone in the outpatient management of patients with low-risk FN, also used this definition of FN.

In conclusion, the present study evaluated the feasibility of outpatient management without initial assessment for febrile patients with breast cancer undergoing adjuvant or neoadjuvant chemotherapy. Although certain guidelines do not recommend outpatient management without initial assessment for febrile patients (4-6), the outpatient management regimen described in the present study was demonstrated to be safe and convenient for febrile patients with early breast cancer. Larger-scale confirmatory studies are warranted to establish guidelines for the management of febrile patients with breast cancer undergoing adjuvant or neoadjuvant chemotherapy.

\section{Acknowledgements}

The authors would like to thank all of the patients who participated in this study.

\section{References}

1. Culakova E, Thota R, Poniewierski MS, Kuderer NM, Wogu AF, Dale DC, Crawford J and Lyman GH: Patterns of chemotherapy-associated toxicity and supportive care in US oncology practice: A nationwide prospective cohort study. Cancer Med 3: 434-444, 2014.

2. Fontanella C, Bolzonello S, Lederer B and Aprile G: Management of breast cancer patients with chemotherapy-induced neutropenia or febrile neutropenia. Breast Care (Basel) 9: 239-245, 2014.

3. Freifeld AG, Bow EJ, Sepkowitz KA, Boeckh MJ, Ito JI, Mullen CA, Raad II, Rolston KV, Young JA and Wingard JR; Infectious Diseases Society of America: Clinical practice guideline for the use of antimicrobial agents in neutropenic patients with cancer: 2010 update by the infectious diseases society of america. Clin Infect Dis 52: e56-e93, 2011.

4. Masaoka T: Evidence-based recommendations for antimicrobial use in febrile neutropenia in Japan: Executive summary. Clin Infect Dis 39 (Suppl 1): S49-S52, 2004.

5. Takamatsu Y: A general description of the clinical guideline for the management of febrile neutropenia. Gan To Kagaku Ryoho 40: 697-702, 2013 (In Japanese).

6. Flowers CR, Seidenfeld J, Bow EJ, Karten C, Gleason C, Hawley DK, Kuderer NM, Langston AA, Marr KA, Rolston KV and Ramsey SD: Antimicrobial prophylaxis and outpatient management of fever and neutropenia in adults treated for malignancy: American Society of Clinical Oncology clinical practice guideline. J Clin Oncol 31: 794-810, 2013.

7. Baden LR, Bensinger W, Angarone M, Casper C, Dubberke ER, Freifeld AG, Garzon R, Greene JN, Greer JP, Ito JI, et al: Prevention and treatment of cancer-related infections. J Natl Compr Canc Netw 10: 1412-1445, 2012.

8. Klastersky J, Paesmans M, Rubenstein EB, Boyer M, Elting L, Feld R, Gallagher J, Herrstedt J, Rapoport B, Rolston K and Talcott $\mathrm{J}$ : The multinational association for supportive care in cancer risk index: A multinational scoring system for identifying low-risk febrile neutropenic cancer patients. J Clin Oncol 18: 3038-3051, 2000.

9. Teuffel O, Ethier MC, Alibhai SM, Beyene J and Sung L: Outpatient management of cancer patients with febrile neutropenia: A systematic review and meta-analysis. Ann Oncol 22: 2358-2365, 2011.

10. Mizuno T, Katsumata N, Mukai H, Shimizu C, Ando M and Watanabe T: The outpatient management of low-risk febrile patients with neutropenia: Risk assessment over the telephone. Support Care Cancer 15: 287-291, 2007.

11. Dulisse B, Li X, Gayle JA, Barron RL, Ernst FR, Rothman KJ Legg JC and Kaye JA: A retrospective study of the clinical and economic burden during hospitalizations among cancer patients with febrile neutropenia. J Med Econ 16: 720-735, 2013.

12. Hendricks AM, Loggers ET and Talcott JA: Costs of home versus inpatient treatment for fever and neutropenia: Analysis of a multicenter randomized trial. J Clin Oncol 29: 3984-3989, 2011.

13. Teuffel O, Cheng S, Ethier MC, Diorio C, Martino J, Mayo C, Wing R, Sung L and Alibhai SM: Health-related quality of life anticipated with different management strategies for febrile neutropenia in adult cancer patients. Support Care Cancer 20: 2755-2764, 2012. 
14. Charlson ME, Pomei P, Alles KL and MacKenzie CR: A new method of classifying prognostic comorbidity in longitudinal studies: development and validation. J Chronic Dis 40: 373-383, 1987

15. Chamilos G, Bamias A, Efstathiou E, Zorzou PM, Kastritis E, Kostis E, Papadimitriou C and Dimopoulos MA: Outpatient treatment of low-risk neutropenic fever in cancer patients using oral moxifloxacin. Cancer 103: 2629-2635, 2005.

16. Sebban C, Dussart S, Fuhrmann C, Ghesquieres H, Rodrigues I, Geoffrois L, Devaux Y, Lancry L, Chvetzoff G, Bachelot T, et al: Oral moxifloxacin or intravenous ceftriaxone for the treatment of low-risk neutropenic fever in cancer patients suitable for early hospital discharge. Support Care Cancer 16: 1017-1023, 2008

17. Rolston KV, Frisbee-Hume SE, Patel S, Manzullo EF and Benjamin RS: Oral moxifloxacin for outpatient treatment of low-risk, febrile neutropenic patients. Support Care Cancer 18: 89-94, 2010.
18. Lyman GH, Dale DC and Crawford J: Incidence and predictors of low dose-intensity in adjuvant breast cancer chemotherapy: A nationwide study of community practices. J Clin Oncol 21: 4524-4531, 2003

19. Griffin DA, Penprase B and Klamerus JF: Relative dose intensity-improving treatment and outcomes in early-stage breast cancer: A retrospective study. Oncol Nurs Forum 39: E459-E467, 2012.

20. Chong Y, Shimoda S, Yakushiji H, Ito Y, Aoki T, Miyamoto T, Kamimura T, Shimono N and Akashi K: Clinical impact of fluoroquinolone-resistant Escherichia coli in the fecal flora of hematological patients with neutropenia and levofloxacin prophylaxis. PLoS One 9: e85210, 2014 